\title{
The impact of the simple orifice schemes of the buffer device in boom refuelling system in the transient pressure of the pipeline
}

\author{
Zhangzhi Dong*, Xiaogang Li, Yanyan Hou, Bin Li, and Yujuan He \\ Air Force Engineering University, Aeronautics Engineering College, 710038 Xi'an, China
}

\begin{abstract}
The boom refuelling system has a large refueling flow, and the refueling joint when the refueling is interrupted in an emergency will cause pressure shock and pressure pulsation. In severe cases, it will cause vibration of the pipeline system, reduce system reliability, and seriously endanger flight safety. Adding a device similar to an accumulator at the end of the pipeline where pressure shock occurs can well absorb the shock pressure. This paper uses a certain type of aircraft as a background to simulate the construction of a rigid air refueling pipeline system with a buffer device. The emphasis is on the pressure shock suppression characteristics of the buffer device. The simulation analysis of the opening parameters of the expansion cavity entrance includes the number and size of the buffer device. The impact of pressure shock performance. The results show that: the more openings and the larger the pore size, the larger the first pressure peak and the smaller the second pressure peak; while increasing the total flow area can effectively reduce the pressure shock suppression effect of the buffer device.
\end{abstract}

\section{Introduction}

The boom refuelling system buffer device is a device used to suppress pressure shock and pressure pulsation. It belongs to the category of pressure pulsation attenuator. The function of this device is mainly to suppress shock and suppress pulsation as a supplement. The buffer device is installed near the front end of the refueling joint and is mainly composed of an air bag, a pressure-resistant shell, and a perforated pipe. It has three states of gas, liquid and solid. The basic structure of the system is shown in Fig. 1. The characteristic of the cushioning device is that it has an airbag structure and a perforated tube structure: the compressibility of the airbag structure in the housing chamber is much greater than that of the oil, so that the chamber can absorb the high pressure oil in the fuel pipeline at the moment of pressure shock, and is capacitive; The tube has multiple rows of small holes, which can consume the energy of high-pressure oil and is resistive. Ideally, the pressure shock generated at the refueling joint will be transmitted to the front end of the buffer device cavity, and oscillate back and forth from the buffer device to the refueling joint until the pulsating pressure is eliminated.

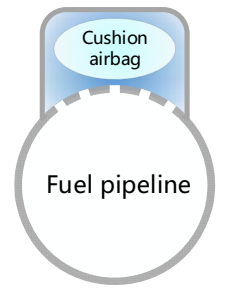

(a) Side view

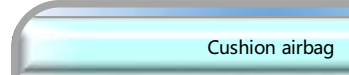

Cushion airbag

Fuel pipeline
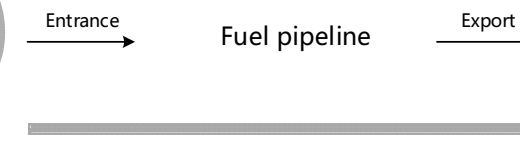

Fig. 1. Schematic diagram of buffer device structure.
Research on the dynamic characteristics of aerial refueling pipeline systems is relatively rare. At the earliest, in 1983, SE Parks of the US Air Force used the transient flow analysis calculation program in the hydraulic system to simulate the transient situation in the pipeline system when the refueling machine and the oil receiving machine of the rigid aerial refueling system were suddenly disconnected. Vibration absorbing equipment with perforated fuel pipeline structure. The fuel enters the cavity of the jacket through the small holes to achieve pressure shock suppression [1]. However, the working pressure of the device is relatively small under working conditions, and it is difficult to absorb large pressure shocks only by small holes and cavities in the pressure shock suppression of the large flow pipeline system. $\mathrm{H}$. W Ng of Nanyang University of Science and Technology in Singapore used Flowmaster software to conduct transient and steadystate analysis of the fuel behavior in the hard fueling system to determine the location where high-pressure vibration may occur, especially the transient process of the pipeline system when the valve is activated [2]. When solving the problem of pressure pulsation induced by fluid transients in the aircraft ground pressure refueling system, Zhou Ruixiang of the Air Force Engineering University and others adopted an active control technology that extracts controllable parameters to monitor the dynamic pressure at the joint Pressure is optimized in real time [3].

In summary, the research on pressure pulsation attenuators in hydraulic systems is abundant, especially in the research of expansion chamber type attenuators, various improvements have been developed, but the

\footnotetext{
* Corresponding author: 1403256991@qq.com
} 
research on pressure pulsation/pressure shock in aircraft piping systems less. Moreover, the research on the rigid aerial refueling pipeline system has been carried out very early abroad, while the domestic start is relatively late [4].

\section{System composition and simulation model}

Fig. 1 shows the basic composition of a certain aircraft hard air refueling pipeline system, which is mainly composed of pipeline system, fuel pump, overflow valve, one-way valve, pipeline valve, pressure regulating valve, vacuum valve, expansion pipe device, Refueling joint composition. The structure of the telescopic tube is shown in Fig. 3.

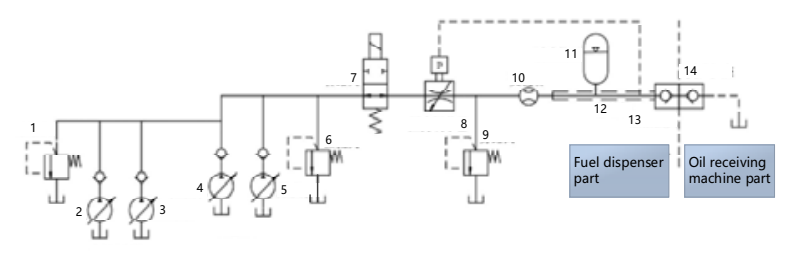

16 9-Relief valve, 234 5-Fuel pump, 7-Pipeline valve, 8Pressure regulator, 10-Vacuum valve, 11-Buffer device, 12flexible tube,13- Refueling connector, 14-Oil socket

Fig. 2. Basic composition of boom refuelling system.

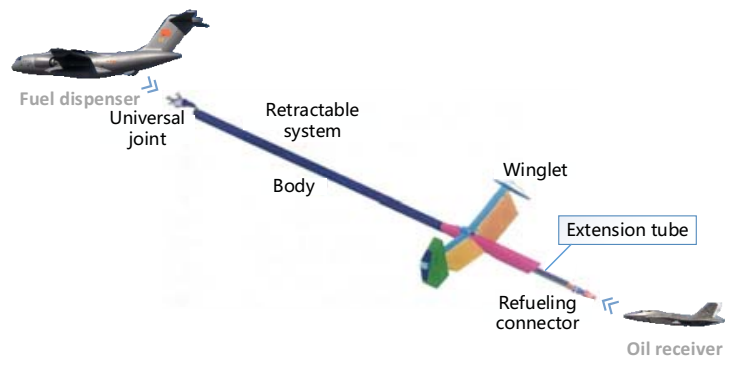

Fig. 3. Schematic diagram of telescopic tube structure.

According to the system schematic diagram in Fig. 2, use AMEsim one-dimensional simulation software to build a system simulation model as shown in Fig. 6 .

\section{Simulation calculation}

There is no specific research and theoretical reference for the design of the inlet size of the expansion cavity of the buffer device, that is, the opening plan of the fueling pipe. However, in the theoretical study of the accumulator, Kong Xiangdong et al. [5] obtained the conclusion that the greater the opening of the accumulator inlet, the better the suppression effect on the pressure shock. The pressure shock suppression effect of the buffer device under the condition of gradually increasing the number and size of openings will be studied.

The number of perforated tubes of the buffer device can be selected from two angles: the number of holes in each row and the total number of rows. One is that the number of holes in each row is unchanged, and the number of holes in each row is changed; the other is that the number of holes in each row is unchanged. Change the number of rows of holes. The openings in the scheme in this chapter are all uniform openings, that is, the opening diameters of the perforated pipes are equal.

\subsection{The total number of rows does not change the effect of the change in the number of holes in each row}

Regarding the structural size of the fueling pipe, keeping the number of perforation sections unchanged, select the number of holes in each row as 1,2,3,4, and 5, with a diameter of $7 \mathrm{~mm}$, and the single row of openings is shown in Fig. 4.

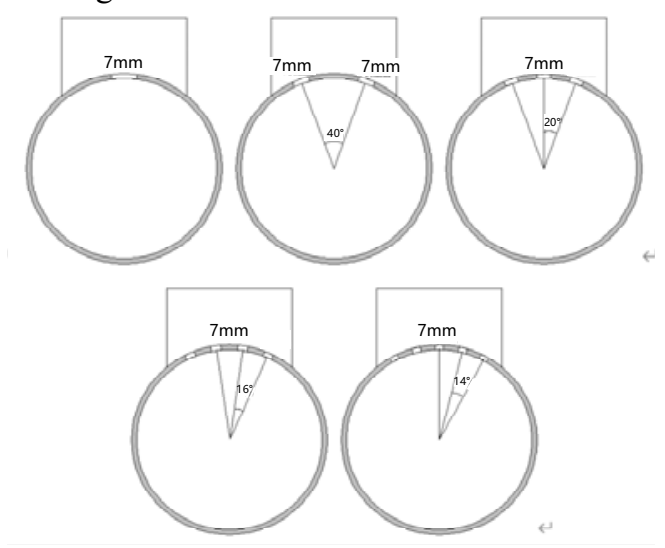

Fig. 4. Single row of holes.

The comparison of pressure changes at the refueling joints when the number of holes in each row is different is calculated as shown in Fig. 5 (the curves 1, 2, 3, 4, and 5 in the figure represent the number of holes in each row as 1, 2, 3, 4, and 5 respectively. The results at time are the same in the following text and will not be repeated).

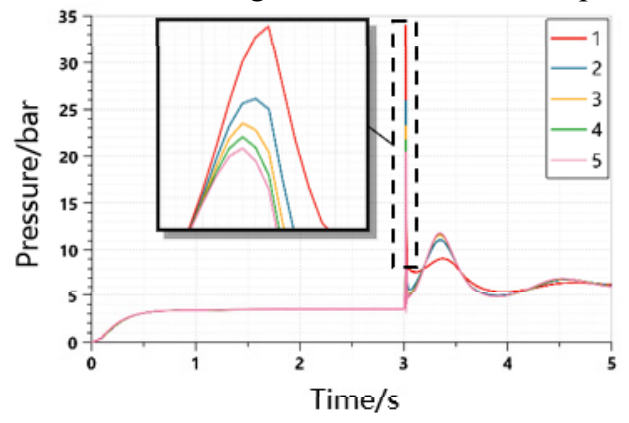

(a) The first pressure peak

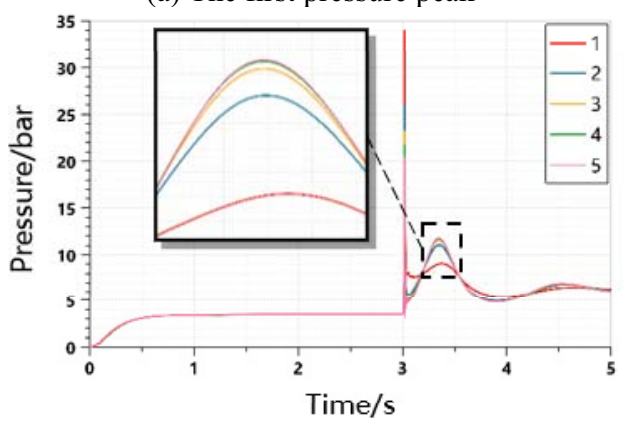

(b) The second peak pressure

Fig. 5. Pressure changes at the refueling joint when the number of holes in each row changes. 


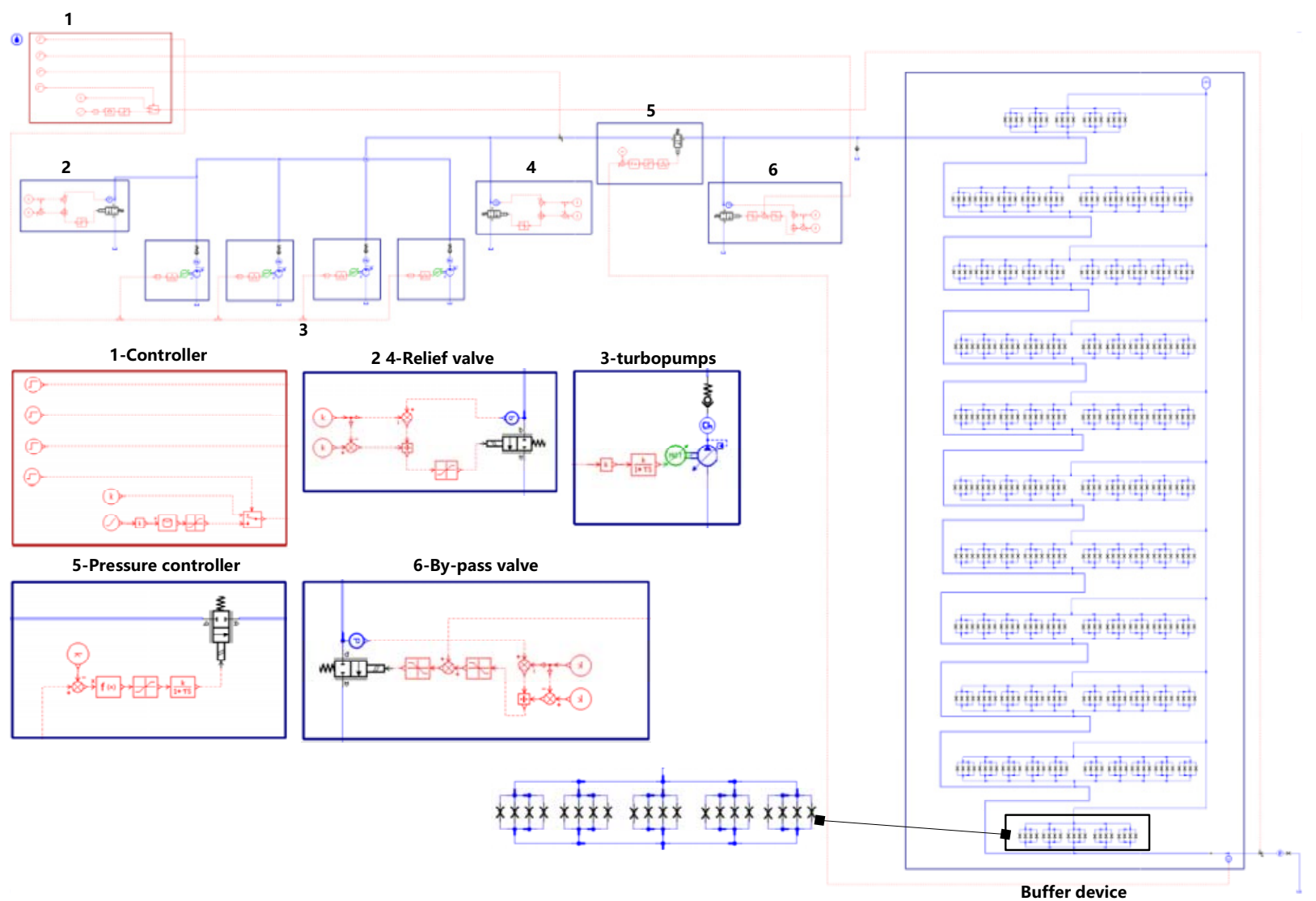

Fig. 6. Schematic diagram of telescopic tube structure.

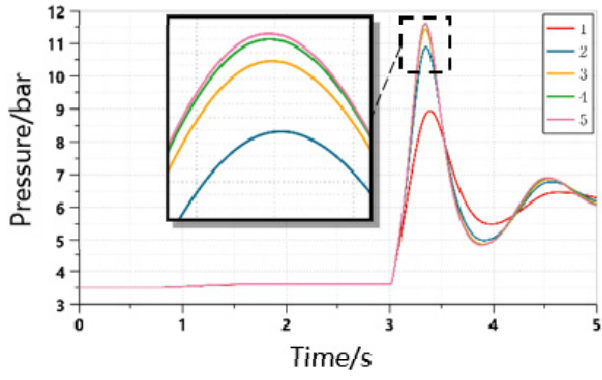

(a) The first pressure peak

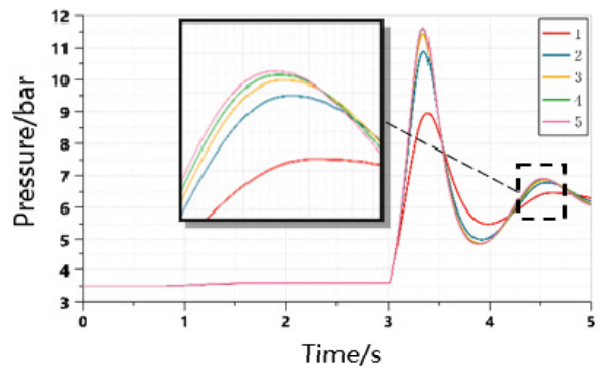

(b) The first pressure peak

Fig. 7. Pressure changes at the refueling joint when the number of holes in each row changes.

It can be seen from the results in Fig. 7 that at the moment of closing the valve at $3 \mathrm{~s}$, an instantaneous impact pressure of up to 33.8 bar appears at the refueling joint, followed by a second pressure peak. The total number of opening rows remains unchanged, with the increase of the number of openings in a single row: the first peak value gradually decreases and the amplitude of hole 1 and hole 2 change the most, and then the amplitude increment gradually decreases; the second time The peak value gradually increases and the increase gradually decreases.

Fig. 7 shows the comparison of the pressure change of the capsule when the number of holes in each row changes. The total number of openings does not change. With the increase of the number of openings in a single row: the gas pressure in the airbag gradually increases, and the peak value of 1 hole changes the most compared to 2 holes. The amplitude increment gradually decreases in the future; the second peak value gradually increases and the increase range gradually decreases.

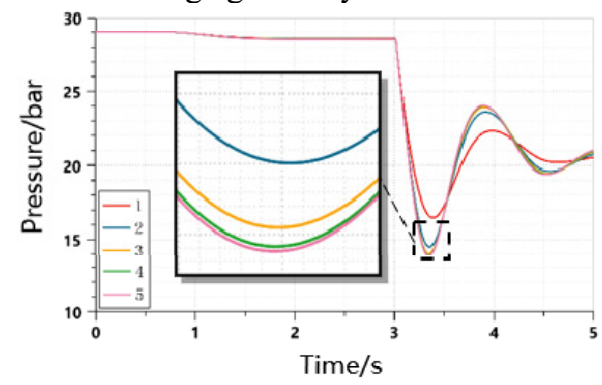

Fig. 8. Capsule volume changes when the number of holes in each row changes.

Fig. 8 shows the change of the capsule volume when the number of holes changes. The total number of openings remains unchanged. With the increase of the number of openings in a single row: the airbag compression volume increases, and the peak value of 1 hole changes the most compared to 2 holes. As the 
number increases, the amplitude increment gradually decreases.
The calculation results of the main parameters are given, as shown in Table 1.

Table 1. Calculation results of main parameters.

\begin{tabular}{ccccc}
\hline $\begin{array}{c}\text { Number of single } \\
\text { row holes }\end{array}$ & \multicolumn{2}{c}{ Impact pressure at refueling joint } & $\begin{array}{c}\text { Maximum airbag } \\
\text { pressure /bar }\end{array}$ & $\begin{array}{c}\text { Minimum airbag } \\
\text { volume /L }\end{array}$ \\
\hline 1 & First peak/bar & Second peak/bar & & \\
2 & 33.8 & 8.8 & 8.9 & 16.6 \\
3 & 25.7 & 10.8 & 10.6 & 14.5 \\
4 & 23.4 & 11.3 & 11.5 & 14.1 \\
5 & 20.9 & 11.5 & 11.7 & 14.2 \\
\hline
\end{tabular}

In summary, the effect of increasing the total number of rows without increasing the number of holes in each row is as follows: the first pressure peak at the refueling joint gradually decreases, and the second pressure peak gradually increases; among them, the peak value of 1 hole has the largest change compared with 2 holes. As the number of holes increases, the peak value gradually decreases.

\subsection{The number of holes in each row does not change. The effect of the change in the number of holes}

Keep the number of openings in a single row unchanged, change the total number of rows of openings, and then change the number of openings. The number of rows of openings is evenly distributed, and the buffer device is selected to have 4 holes in each row with a diameter of $6 \mathrm{~mm}$, as shown in Fig. 9.

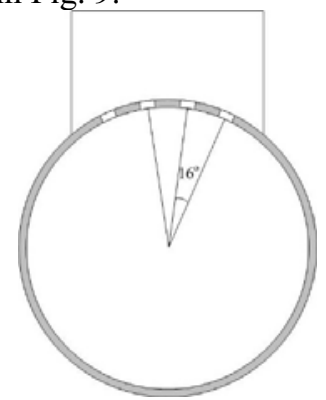

Fig. 9. Buffer device opening situation.

Select the number of rows of openings as: 51 rows, 61 rows, 73 rows, 81 rows, 91 rows, then the node spacing.

Fig. 10 shows the comparison of pressure changes at the refueling joint when the number of rows of openings are different. As a result, the following text is the same and will not be repeated.

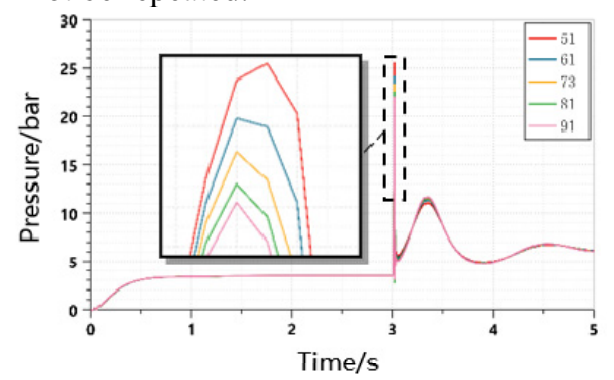

Fig. 10. Buffer device opening situation.

From the results in Fig. 10, it can be seen that at the moment of closing the valve at $3 \mathrm{~s}$, an instantaneous impact pressure of up to 25.8bar appears at the refueling joint, followed by a second pressure peak. The number of holes in each row remains the same. With the increase of the total number of rows: the first peak value gradually decreases and the decrease amplitude also gradually decreases, that is, the suppression effect of pressure shock is gradually reduced by increasing the number of holes; the second time. The peak value gradually increased and the increase rate gradually decreased. The simulation calculation results are shown in Table 2.

In summary, the effect of the increase in the number of holes without changing the number of holes is as follows: the first pressure peak at the refueling joint is gradually smaller, and the second pressure peak gradually increases; among them, the peak change of 51 rows is the largest compared to 61 rows, only $1.6 \mathrm{bar}$, the peak value gradually decreases in the future.

Table 2. Calculation results of main parameters.

\begin{tabular}{ccccc}
\hline $\begin{array}{c}\text { Number } \\
\text { of rows }\end{array}$ & $\begin{array}{c}\text { Impact pressure at } \\
\text { refueling joint } \\
\text { First } \\
\text { peak/bar }\end{array}$ & $\begin{array}{c}\text { Second } \\
\text { peak/bar }\end{array}$ & $\begin{array}{c}\text { Maximum } \\
\text { airbag } \\
\text { pressure } \\
\text { /bar }\end{array}$ & $\begin{array}{c}\text { Minimum } \\
\text { airbag } \\
\text { volume /L }\end{array}$ \\
\hline 51 & 25.8 & 11.1 & 10.9 & 14.3 \\
61 & 24.2 & 11.4 & 11.4 & 14.2 \\
73 & 23.3 & 11.6 & 11.6 & 14.1 \\
81 & 22.5 & 11.7 & 11.7 & 14.0 \\
91 & 22.2 & 11.8 & 11.8 & 13.9 \\
\hline
\end{tabular}




\section{Conclusion}

This paper simulates and analyzes the impact of the buffer device on pressure shock suppression from the perspective of the number of openings and the size of the openings when the total flow area of the buffer device inlet changes. The main conclusions are as follows:

(1) Keep the total number of rows constant Change, the more holes in each row: the smaller the first pressure peak at the refueling joint, the greater the second pressure peak; among them, the peak value of 1 hole has the largest change compared with 2 holes, and the peak value will gradually decrease afterwards.

(2) Keep the number of holes in each row unchanged, and the more rows of openings: the smaller the first pressure peak at the refueling joint, the greater the second pressure peak; the 51st row has the largest change from the 61st row, and the peak gradually decreases thereafter.

(3) Under the condition of meeting the requirements of strength and installation space, increasing the number of openings can greatly improve the suppression effect of the device on pressure shock, and the increase in the effect will gradually decrease as the number of holes increases.

\section{References}

1. Parks, S. E., and M. E. Franke et al.,. "Transient flow analysis of an aircraft refueling system", $J$. Aircraft., vol. 20, no. 12, pp. 1033-1036, 1971.

2. H. W. Ng, and F. L. Tan, "Simulation of fuel behaviour during aircraft in-flight refueling", Aircr. Eng. Aerosp. Tec., vol. 81, no. 2, pp. 99-105, 2009.

3. R.-X. Zhou, et al., "Aircraft Pressure Refueling Pipeline Dynamic Pressure Control Technology", Journal of Air Force Engineering University.(Natural Science Edition), vol. 5, pp. 1-3, 2003.

4. J.-W. Tian, "Analysis of the development of China's aerial tanker", Tank and armored vehicles, vol. 20, pp. 20-24, 2016. doi:10.19486/j.cnki.111936/tj.2016.20.008.

5. X.-D. Kong, X.-X. Zhu, L.-X. Quan et al., "Simulation study of pressure shock absorption by proportional valve-controlled accumulator", Machine tools and hydraulics, vol. 11, pp. 131134+81, 2018. 